\title{
Strategie per l'incremento della percentuale di pazienti uremici in dialisi peritoneale in Italia
}

\author{
Giovambattista Virga, Stefania Mastrosimone
}

\author{
Servizio di Nefrologia e Dialisi, Ospedale di Camposampiero, Padova
}

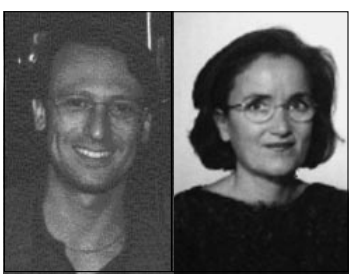

Giovambattista Virga e Stefania Mastrosimone


p a z i e n t i dializzati, in Italia, è stabile attorno a un valore del $10 \%$ mentre a livello mondiale si assesta sul $15 \%$.

L'incremento nell'uso della PD in Italia è auspicabile per il minor costo della metodica, per il rischio di sovraffollamento dei Centri dialisi e per aumentare l'opportunità per i pazienti di usufruire di una metodica che rallenta maggiormente la perdita della funzione renale residua rispetto all'emodialisi (ED).

Le ragioni del mancato incremento percentuale dei pazienti in terapia con PD, in Italia, sono molteplici ma possono essere suddivisi in due fondamentali:

A. elevato numero di Centri dove la $\mathrm{PD}$ non è praticata;

B. scarso aumento della percentuale dei pazienti in PD nei Centri dove è praticata.

A. L'elevato numero dei Centri che non praticano la PD è verosimil- mente dovuto a fattori quali:

A1. l'entità del rimborso, inferiore alla ED;

A2. la mancata crescita di una cultura nefrologica che considera utile l'inizio di un programma di PD;

A3. la difficoltà nell'allestimento di strutture necessarie per la PD (locali e personale dedicati).

B. Lo scarso aumento percentuale dei pazienti trattati con PD nei Centri dove è praticata è dovuto a fattori quali:

B1. la mancata crescita di una cultura nefrologica che considera la PD una metodica dialitica con risultati sovrapponibili all'ED (almeno nei primi 3 anni);

B2. l'aumento dell'età anagrafica media dei pazienti che riduce percentualmente quelli in grado di autogestirsi;

B3. l'assenza di criteri univoci riguardo le controindicazioni cliniche alla PD;

B4. l'opinione generale dei pazienti uremici sulla PD non sempre coerente con la realtà;

B5. la difficoltà nel mantenere $\mathrm{i}$ pazienti in PD ("patient retention").

Ecco le strategie possibili.
A1. Unificare il rimborso per il trattamento dialitico, così che sia interesse della Struttura sanitaria proporre al paziente, quando possibile, il trattamento più economico.

A2. Diffondere tra i nefrologi la convinzione che l'allestimento di un programma di PD permetterebbe di fronteggiare con più rapidità e minori costi l'incremento delle immissioni in dialisi anche per evitare costose ristrutturazioni di Centri dialisi, ormai saturi, e garantire con minore personale infermieristico la gestione di un maggior numero di pazienti.

A3. Convincere le Unità amministrative sull'opportunità di creare spazi idonei e di addestrare personale infermieristico dedicato con un investimento iniziale per un rendimento nel medio-lungo termine.

B1. Motivare i nefrologi dei Centri dove è presente un programma di PD e dove la PD è spesso considerata metodica di seconda scelta da riservare ai pazienti più problematici affinché non eseguano una selezione in negativo dei pazienti o nessuna selezione, contribuendo a creare una immagine negativa della metodica. 
B2a. Allestire un protocollo rigoroso ed esteso a tutti i pazienti uremici per la valutazione della loro idoneità alla PD al fine di evitare che soggetti potenzialmente adatti alla PD vengano trattati con ED prima del giudizio del personale incaricato;

B2b. immettere, da subito, il paziente giovane in lista per trapianto al fine di garantire il più possibile un programma di terapia sostitutiva costituito solo da PD e trapianto con il vantaggio di sfruttare al meglio le potenzialità della metodica evitando di subirne i limiti;

B2c. incrementare, dove è presente un supporto familiare disponibile, l'uso della APD nei pazienti anziani al fine di ottenere, dove necessaria, una collaborazione familiare per un idoneo periodo di tempo.

B3. Uniformare, tra i nefrologi dei Centri dove è presente un programma di PD, i criteri relativi alle reali controindicazioni alla PD.

B4. Fornire i Centri, dove è presente un programma di PD, di mezzi audiovisivi da utilizzare per i pazienti al fine di rendere la scelta della metodica legata ad aspetti più realistici possibile.

B5. Selezionare i pazienti per la PD tra quelli idonei e motivati al fine di aumentare la "patient retention" oltre a migliorare l'immagine della metodica. A tal fine è indispensabile una informazione, rivolta al paziente, specialistica, completa ed esaustiva riguardo tutti gli aspetti della metodica. Inoltre, poiché le capacità professionali del personale medico ed infermieristico dedicato alla PD giocano un ruolo fondamentale nel successo del programma, sono indispensabili un investimento formativo adeguato e una autonomia gestionale.
Nella nostra esperienza, dall'inizio del programma di PD ad oggi (5 anni e 10 mesi), abbiamo seguito costantemente la strategia di utilizzare la PD solo nei pazienti idonei, motivati e volontari. Abbiamo quindi eseguito una selezione "in positivo" che ha portato comunque a un costante incremento dei pazienti in PD, sia in numero assoluto (30) che in percentuale $(26 \%)$. Il range di età anagrafica dei nostri pazienti (20-85 anni) dimostra che la selezione è stata effettivamente eseguita solo riguardo l'idoneità e la motivazione. Non abbiamo considerato altre indicazioni e controindicazioni, eccetto la presenza di stomie addominali. Pochissimi pazienti sono sfuggiti al protocollo di valutazione per l'idoneità alla PD.

I dati riguardo la sopravvivenza dei pazienti, il tasso di peritoniti e la "patient retention" sono soddisfacenti. Il drop-out per motivi di burn-out del paziente si è verificato in un solo caso. L'immagine della PD nel nostro Centro è buona sia tra i medici che tra gli infermieri oltre che tra $\mathrm{i}$ pazienti. Tutto questo facilita l'ingresso volontario dei nuovi pazienti che, motivati, contribuiscono al successo e all'immagine della tecnica creando un circolo virtuoso.

Il nostro programma ha potuto usufruire, sin dall'inizio, di locali e personale dedicato, con un investimento che si è rivelato fruttuoso. Se i pazienti attualmente in PD fossero tutti trattati con l'ED la capacità del nostro Centro sarebbe largamente sopraffatta.

In conclusione, la nostra opinione è che una percentuale di pazienti trattati con PD in Italia, almeno doppia di quella attuale, sarebbe raggiungibile a condizione che tutti i Centri dialisi elaborino un programma di PD che preveda personale e locali dedicati, una valutazione di idoneità tempestiva ed estesa a tutti i pazienti uremici e una selezione dei pazienti più adatti e motivati. Il rimborso per i pazienti dializzati andrebbe unificato su una cifra media che renda vantaggiosa la PD. 